Rapid Reviews COVID-19

\title{
Reviews of "Information Delivered by a Chatbot Has a Positive Impact on COVID-19 Vaccines Attitudes and Intentions"
}

Tom Stafford ${ }^{1}$, Charlotte O. Brand, Pierre Verger ${ }^{2}$

${ }^{1}$ The University of Sheffield Department of Psychology, UK,

2Observatoire Regional de la Sante Provence-Alpes-Cote d 'Azur: Observatoire Regional de la Sante Provence-Alpes-Cote d'Azur, Research Department, Faculté des sciences médicales et paramédicales, France

Published on: Mar 07, 2021

License: Creative Commons Attribution 4.0 International License (CC-BY 4.0). 
To read the original manuscript, click the link above.

Summary of Reviews: Amidst growing concerns about widespread vaccine hesitancy, this preprint offers important insights into a possible scalable intervention. Reviewers suggest further investigating the mechanisms at play in the chatbots effectiveness.

Reviewer 1 (Tom Stafford, Charlotte O. Brand)

Reviewer 2 (Pierre Verger)

\section{RR:C19 Strength of Evidence Scale Key}

$$
\begin{aligned}
& \text { प } \\
& \text { प्र } 40 \square=\text { Not Informative } \\
& \text { प्रा पि = Potentially Informative }
\end{aligned}
$$

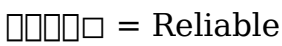

$$
\begin{aligned}
& \text { प्राप्र = Strong }
\end{aligned}
$$

To read the reviews, click the links below. 